ANTIPODE SPECIAL ISSUE - INTRODUCTION

The Social Life of Robots: The Politics of Algorithmic Life, Governance, and Sovereignty

Del Casino Jr., V. ${ }^{1}$, House-Peters, L. ${ }^{2}$, Crampton, J. $^{3}$, and Gerhardt, H. ${ }^{4}$

Introduction

This special issue emerges out of an ongoing set of conversations amongst geographers who have been increasingly thinking about how human and more-than-human relations with robots and robotic technologies are reworking the sociospatial dimensions of our lives. This special issue takes one cut at this concern. The four papers included explore how the rapidly changing, and increasingly networked, world of robots and robotic technology development is shifting and disrupting geographic imaginaries and everyday social, cultural, and ecological practices. Here, the terrain of robots and robotics is interpreted broadly to consist of the hardware and software that can be found in the materialities of robot bodies, and the algorithmic logics and machine learning capacities of new emerging digital technologies. Geographers have produced groundbreaking work interrogating what robots and robotic technologies mean for discipline, surveillance, and security in the 21st century (e.g., Amoore and Raley 2016), and how these technologies may "travel" from hubs to sites of application (e.g., McDuie-Ra and Gulson 2019). This special issue takes as its point of departure the role of these technologies and their associated materialities in making and remaking the structures, conditions, and relations of everyday life.

It is important to note that this body of work on robots and robotic technologies is partially related to but also parallels the recent wave of attention to and growth of geographic research produced through, by, and of the digital, what Ash, Kitchin, and Leszczynski (2018) have termed a critical 'digital turn' in geography. This turn has focused on questions of smart cities (Datta 2015), digital media and communication (Adams 2017), the security state (Shaw 2013, 2016, 2017), and the automation of environmental conservation (Arts et al. 2015; Adams 2017), writ large. It does less, though, to think through the reimagination of human-nonhuman relations, subjectivities, and potentialities that come to be possible in a world already populated by robotic possibilities. It also shies away from fully interrogating the ways in which these relations are altering meanings of the concept of human intelligence or cognition (Lynch and Del Casino 2019).

\footnotetext{
1 Professor, Department of Urban and Regional Planning, San José State University, vincent.delcasino@sjsu.edu (contact author)

2 Assistant Professor, Department of Geography, California State University, Long Beach, lily.housepeters@csulb.edu

3 Professor, School of Architecture, Planning and Landscape, Newcastle University, jeremy.crampton@ncl.ac.uk

4 Professor, Department of Geosciences, University of West Georgia, hgerhard@westga.edu
} 
That is why a turn to robotics - the spaces of algorithmic logic (Crampton 2016), the capacity of "the cloud" to manage geopolitical worlds (Amoore 2018), the impact automation may have on work and life (Richardson and Bissell 2017; also see Richardson this issue), and more generally the meaning of robots and robotic systems within the confines of critical urban studies in human geography (Macrorie, Marvin, and While 2019) and subfields, such as political ecology (e.g., Robbins 2017), which has only just begun to engage with robots and robotics - is so important. Thus, in focusing on robotics as a component of much more complex human-nonhuman relations a number of critical questions emerge: What sorts of human and nonhuman subjectivities are made possible and/or closed off by the rise of new robots and robotic technologies? What are the attendant anxieties around automation and algorithms and their failures, gaps or uncertainties? What role might robots play in our understanding of the spatialities of key concepts in human geography, including labor and labor politics, war and warfare, health and health care, or leisure and entertainment, to name a few?

Underlying all of these questions is the constant reshaping and shifting of power relations and the emergence of new forms of domination, as well as resistance. As advances in robots and robotic technologies create ever more capacious, ubiquitous, and networked machines, and as the desires for and dependencies on these technologies creep into the most intimate spaces of human life and decision-making, it is crucial to consider the changing landscape of power, and subsequent resistance, that is emerging (see Crampton 2019). The proliferation of code into everyday life serves to produce and reproduce novel space-time configurations and imaginaries (Cockayne and Richardson 2017, 2019) and attendant processes of regulation, differentiation, disruption, and transgression. As Lupton (2018) argues data come to matter through the everyday, embodied encounters between bodies and digital technologies that serve to render comprehensible bodily processes, vitalities, and desires in the digital realm. At the site of articulation of the body and big data, the abundance of digital tracking systems and the growing reliance on, habituation to, and acceptance of this form of data production and surveillance of everyday routines serves to transmogrify data from exceptional to mundane (Pink et al. 2017).

As Amoore (2019) rightly points out, however, within the confines of this world of machine learning and algorithmic life lie spaces of subject centered doubt and uncertainty that manifest in these emerging relations of humans and nonhumans. As she argues: "This doubtful subject is not recognizable as a unitary individual but is a composite subject in whom the doubts of human and non-human beings dwell together, opening onto an undecidable future, where one is permitted to ask new questions on the political landscape" (149). In de-centering the subject in this way, within the confines of the algorithmic life produced through artificial intelligence and machine learning, we might better resituate our own systems of intelligence, cognition, and knowing in new forms of posthuman doubt. It is here, then, at the intersection of the robotic, the 
human, and more-than-human nature that we may begin to rethink the new geographies emerging before us.

\section{The "Rise of the Robots"}

Robots are often imagined as material objects with bodies and form, yet they are also invoked in software, code, and algorithms (Del Casino 2016). This is not to suggest an either/or ontology of robots but a both/and whereby geographers must take up the theoretical and political implications of the hardware/software matrix and what it means for human and more-thanhuman bodies and relations. Assemblage theory and other theories of power and performance that cut across the logics of representation and non-representation empirically and theoretically interrogate emerging robotic futures, human cyborg relations, and other robotic possibilities. There are a number of critical developments that come to mind here, for instance, automation in manufacturing settings (Bissell and Del Casino 2017), the emergence and potential of 3-D printing in education (Oswald, Rinner, and Robinson 2019), and the increasing role of algorithmic objects in making and influencing decisions in fields across urban planning (Batty 2013), policing (Linder 2019), finance (Kear 2017), social media (Gieseking 2018), and environmental management (Bakker and Ritts 2018; also see Lockhart and Marvin this issue).

Related to the above concerns, robots are also transforming the spaces, politics, and subjects of security (e.g., Shaw 2013, 2016, 2017). From biometric borders, automated gun turrets to mobile sea mines, a new class of robotic apparatuses are being developed, each of which embodies (and mobilizes) a future geography. The rise of U.S. drone warfare and policing has already received a great deal of media and academic discussion but there is a whole host of current and potential robotic automation in the military (and eventually the police) that is harkening the end of warfare (and policing) as we know it, not only in terms of how it is conducted but in terms of the aims that can be achieved. The ultimate question here becomes how robots will transform not only the spaces of war and conflict, but the context of geopolitical calculation and engagement (c.f., Boyce 2016). Related to this is the question of how the geographical dimensions of resistance to the state and statecraft will be affected and whether robots do or could play a role in such resistance.

Paradoxically, the focus on the military and police has tended to mask the wider robotic revolution in security: the banal and everyday deployment of robots by state and non-state actors employed to track, analyze, and store all sorts of information on people, and the counter use of robots and robotics to circumvent these efforts, construct new networks of technological solidarity and sovereignty, or repurpose them for criminal purposes (see Lynch this issue). When considering these ubiquitous and far reaching changes, geographers will need to ask how the spaces and architectures of policing, surveillance, and securitization will be transformed and 
what potentials exist, or newly emerge, for resistance and subversion? Geographers are wellpositioned to explicate how these changes will be realized differently across different spaces and vis-a-vis different bodies, and how spaces will develop differently as a result. How does technology such as robots and machine learning "travel" from hubs of production such as China and Silicon Valley to sites of application (McDuie-Ra and Gulson 2019)? As Faroohar (2019) documents, although divided by political philosophy, these locations are engaged in mutual coproduction, with western technology seeking entry to China and vice versa, so that for example surveillance technologies boomerang back to the west via China.

Advances in sensing technology and robotic system design increasingly target the more-thanhuman environment producing new encounters with and understandings of nature (see Lockhart and Marvin this issue). Environmental monitoring is increasingly carried out via complex, autonomous, and networked sensor systems, mobile robotic platforms, and UAVs/drones. The articulation of digital technology, big data, and nature conservation has drawn new alliances between artificial intelligence, machine learning, automation, and the management of endangered wildlife and sensitive habitats, branded digital conservation (Arts et al. 2015) and conservation by algorithm (Adams 2017). The ability of these systems to collect and wirelessly transmit data at continuous time scales, reach remote locations, and carry out panoramic measurements is shifting the temporal and spatial dimensions of environmental perception and political possibility (Lehman 2016).

While big environmental data sets present new opportunities for sense-making in opaque and unwieldy environments, such as the ocean, the growing emphasis on algorithms and machine learning is transforming not only how we claim to know nature, but the very kinds of interventions, infrastructures, regimes of care, and resource governance modalities that become possible (Lehman 2018; Boucquey et al. 2019; Gabrys 2019). Big data and algorithmic governance have also infiltrated a site of more commonplace human-environment relations, the farm. Here, far the smart city, automation, machine learning, and precision technology are transforming rural landscapes and relations, as agriculture becomes a new site for surveillance (Klauser 2018) and non-human animal bodies enter into and adjust to novel relations with robots (see Holloway 2007 for a discussion of bovine subjectivity and robotic milking technology). As humanenvironment relations become increasingly mediated by and refracted through robots and robotic technologies there is a need to consider technical glitches and slippages, where the unexpected and unanticipated arises, and the potentials positive and negative that they enable (Leszczynski 2019). Yet, attention to algorithmic human-environment relations remains limited. Given this lacuna, there is a need for geographers to examine and theorize how the rise of robots are reorganizing ways of knowing, seeing, producing, and intervening in nature, and the political economic and environmental justice implications of this reorganization. 
Algorithms, Governance, and Sovereignty

Along with the rise of the robots comes a set of core questions geographers have begun to ask around the issue of algorithms and algorithmic life, geopolitical governance, and the future of sovereignty, be that political or technological. Each of these topics are, in and of themselves, unique objects of inquiry. For the purposes of this introduction, however, and as a way to frame the papers that follow, we open up a few core questions raised through the discussion of each of these objects of study within the confines of robots and robotic technologies, particularly as they relate to the complicated social, human, and more-than-human geographies of everyday life.

As we are growingly aware, robots and robotic technologies are today empowered by the evolution of algorithmic work that allows for an increasing look at the micro at the expense of the macro. This almost hyper-inductive world of new "facts" is generated by bits and bytes of data smashed together and parsed through algorithms both humanly created and artificially generated through machine learning technologies. As Tarleton Gillespie (2014) defines them, algorithms are a way of taking an input and performing a calculative task in order to produce a desired output. This definition usefully foregrounds the notion of desire, and thus, implicitly, the human, although it need not necessarily preclude the machinic possibilities enabled by our robotic futures. Put another way, we are often discussing human "intelligence augmented" (IA) as much as we are discussing "artificial intelligence" (AI) (see Lynch and Del Casino 2019).

That said, we should also ask what work algorithms do once they "arrive" at their site of application. To some extent, this is a question of what values are being encoded into the algorithms in the first place. Some shift of perspective is required here. Principles of "fairness, accountability and transparency (FAT) or "responsible Al" such as the recent Montreal Declaration look to assess the outcomes of Al and machinic infrastructures with a view to preclude undesirable outcomes such as "killer robots." However, the values that are encoded into them depend to some extent on the imaginaries that frame the algorithms, whether this be security, efficiency, profit or social justice. These imaginaries constitute a digital and material infrastructure, which as Ruha Benjamin has observed, constitute a "new Jim Code" or carceral technoscience, a racist and racializing imperative (Benjamin, 2019). As Langdon Winner (1980) observed, artifacts have politics. Here too is the perspectival shift; it is not technology that is the cause of injustice, rather, technology is the symptom. Treating the symptom will just result in the production of new symptoms. It is the infrastructure itself and its imaginaries that are racist, patriarchal, homophobic, exploitative, etc. As such, many people are forced to live inside other people's fantasies. In the new Jim Code, "race neutrality" is more damaging than racial bias, because the former allows unwitting racial discrimination. The point of activist intervention 
therefore would be to develop new "abolitionist imaginaries" that work not at the site of the symptom--the technology of the algorithm or robot--but in the encoding of value.

As many scholars also note, then, the rise of algorithmic life - a life managed in relation to the explosion of algorithmic fantasies and projections put in front of us, as humans, by organizations, such as Amazon - has also raised critical questions related to the geographies of governance and "the manifold ways that algorithms and code/space enable practices of governance that ascribes risk, suspicion and positive value in geographic contexts" (Crampton and Miller 2017, n.p.). Algorithmic governance is an assemblage of human and non-human actors, both material and discursive. Any history would therefore have to disentangle algorithms' instantiations in digital technologies from what Andrea Miller (2017) calls their broader protocological capacities. These protocological capacities might emerge materially or immaterially, taking digital, non-digital, and not-only-digital forms in databases, museums, and archives, state and corporate infrastructural apparatuses, and colonial logics of population management and policing. For Antoinette Rouvroy and Thomas Berns (2013), as the algorithm's concern with relational data supersedes human decision-making and its attendant forms of knowledge production, it ushers in a form of governance that is not immediately legible as politics as such. For Rouvray and Berns, algorithmic governance comprises three "stages":

1. The generation of the data double and Big Data, e.g. facial movements become statistical data (cf. Deleuze 1992). Here, data are "accumulated by dispossession" (Harvey 2003), an extraction but also a substitution, a standing-in-for or representation.

2. Automated knowledge production. In this stage, marked by machine learning and absolute objectivity, the databases are acquired and formed.

3. Action on behaviours. Anticipate and preempt individual desires and behavior and associate with profiles (Rouvroy and Berns 2013)

Drawing from Deleuze and Guattari, Rouvroy and Berns identify algorithmic governance as rhizomatic. In this sense, to what degree can we analyze algorithmic governance as a new form of digital infrastructure? Here we do not identify a static structure, but one that is characterized by its emergent qualities, across which automated decision-making and machinic learning can "travel" from centers of expertise and production (Silicon Valley, China) to sites of application and consumption (school classrooms, police bodycams, or facial recognition technologies for surveillance of urban protests). Tracing how Al travels is thus an urgent task in understanding how automated decision-making, a critical feature of robotics, acts to circumscribe personal and even institutional sovereignty. In the latter, we might refer to judges being bound by algorithmic scoring protocols at sentencing, or universities being ham-strung by financial models that are so precisely tuned as to deny them agency in protecting employee pensions. 
From the above, a broad takeaway is that algorithmic robotic technologies are increasingly becoming woven into, and thus helping to create, our complex, continuously evolving, and contingent socio-spatial realities. This technology, and the data it consumes, is created and employed to achieve particular ends (economic, security), and in turn, it demonstrates its own agency, leading to unexpected outcomes, challenges, and opportunities. This multi-faced encounter of human-robotic interactions is thus saturated with specific power relations and subsequent entry points to critical engagement, subversion, and resistance. That is why robots can be seen as furthering the interests of the powerful. Big Tech, which presents itself as an alternative capitalist force of good, is enabling a new cycle of capital accumulation, thus extending capitalism's run even further. The extraction of "free" data and its manipulation is creating new efficiencies and markets for profit - the selling of Facebook data and its algorithmic deployment to shape the 2016 U.S. election is a good example. At the same time, this technology is employed to enhance the power of control, and even repression, of the state. Silicon Valley's self-presentation, encapsulated in Google's code of conduct phrase, "Don't be evil", which has since morphed into Alphabet's "Do the right thing", can certainly be debunked on multiple fronts (Faroohar 2019).

Yet, concomitantly and paradoxically the rise of the robots, as the digital turn more generally, poses risks to the powerful. This is well captured in the oft cited expression, "information wants to be free" (Brand 1985:49). While information (data and code) are increasingly valuable in our contemporary political economic system, it is also very difficult to contain. The practically infinite reproducibility of digital content is therefore seen by some as an existential threat to large swathes of the economic system (Bauwens 2005; Mason 2015; Rifkin 2014). Whether it's the music files of old or emerging complex Al systems, there is no physical boundary beyond the infrastructure and energy supporting this content that prevents universal sharing. As a consequence, there emerges a clear struggle over the containment of digital value, with large corporate IT interests working to create centralized platforms acting as digital content gatekeepers, and a variety of hacktivists seeking to circumvent and ultimately destroy these platforms in favor of decentralized information (Greenberg 2012; Kleiner 2010).

In a similar vein there are efforts to reclaim the actual data that feeds the emerging Al systems. Cypherpunk hacktivists are using cryptographic technology to not only block the corporate collection of data, but also the surveillance of the state. Blockchain is here pointed to as a quickly evolving cryptographic technology based on an organizing principle of cooperative decentralization, which can be applied, for example, to data storage and Al capacity, as opposed to a corporately controlled, centralized cloud (Zook 2018). Various crypo-currencies are also emerging using blockchain technology as a way to fundamentally undermine the monopoly of value maintained by state sanctioned money, thus potentially undermining both capitalism and the state in one go (Zook and Blankenship 2018; see Gerhardt this issue). 
Indeed, the struggle over the digital - including data, code, and processed information - is increasingly framed as a struggle over a new, digital commons. The digital commons are here viewed as being collaboratively created and governed for the good of the whole. The capitalist values of competition, enclosure, and profit are thus countered with cooperation, commoning, and sharing as is evidenced by already existing and quite extensive collaborative, voluntary production that is made available as open sourced value. There are myriad examples of this kind of peer-to-peer (P2P) production and the crowdsourcing and sharing of data, from Wikipedia and Apache software to OpenStreetMap, as the best geographical example (McConchie 2015; Sieber et al. 2016). Together this impetus is leading to the growth of technological sovereignty movements in cities, such as Barcelona (see Lynch this issue).

Of course, the challenge to these counter-power activities is that they often remain partially regulated by the larger political economic structures in play while they also risk becoming coopeted. Voluntary P2P activity won't pay the bills and open-sourced code and data, offered in the spirit of a digital commons, is regularly pilfered and profited off of by companies who eagerly use the unremunerated labor (Wilson and Kleiner 2013). It is in response to this challenge that demands for technological sovereignty are made. Such demands include municipalizing the control of internet infrastructure, de-privatizing and making big data open access, transferring the digital commons to the material through public maker spaces and fab-labs, ensuring net neutrality, easing intellectual property and patent laws, developing reciprocity measures for when the digital commons is used for profit, and supporting a guaranteed income for all (Bauwens et al. 2019).

Although machinic objects and human-machine interactions have long been recognized as capable of reordering and rescripting social relations, political fields, affective states, and ecological encounters - as well as being seen as central to the production, organization, and performance of state power - this special issue interrogates how robots engender new sets of questions ripe for geographic analysis. In turning toward robot(ic)s, the included papers confront shifting terrain in relation to major geographic themes: life, labor, nature, security, sovereignty, resistance, and social change.

\section{Contributions of the Special Issue Papers}

The four papers included in this special issue take our present moment of algorithmic life as a starting point for contemplating how human and more-than-human relations with robots and robotic technologies are reworking the sociospatial dimensions of our lives through attention to the possibilities, tensions, and limits presented by automation and algorithmic governance. The authors present nuanced and innovative interpretations of robotics as integral and transformative, yet uncertain and contingent, components of the complex human and morethan-human relations that structure our modern world. 
Richardson's paper elucidates a facet of everyday algorithmic life through an intimate analysis of how socio-economic arrangements come to produce and be produced through the automated coordination of food delivery. An auto-ethnographic examination of the UK food delivery company Deliveroo is used to show how technological platforms are more than just interface and algorithm as they give rise to, and become part of, specific socio-spatial and temporal configurations. Richardson argues that the market that the Deliveroo platform is helping to create is doing more than simply mediating supply and demand. Rather, the market is a form of "agencement," constantly made and remade via the many contingent interactions of the various moving parts. In this sense the platform makes up a critical element within an emergent, flexible market that enables and constrains subject interactions, which, in turn, results in new geographical expressions.

By decentering the interface and seeking to widen the lens of analysis, Richardson focuses attention on the processes of making calculable each step and interaction of a Deliveroo journey. The Deliveroo platform is posited as a component of a larger, more complex articulation of various subjects and objects, including customers, restaurant workers, drivers, and the actual food. Here the dynamics of calculation, flexibility, and contingency come into direct relation, and at times conflict, with the accelerated temporalities of "on-demand" consumption culture. Richardson's analysis illustrates how processes of calculation produce contingency, rather than resolving it, revealing how contingencies are inherent to the algorithmic calculation of goods and services via markets negotiated through platform technology. Attending to points of interaction conducted by specific human and more-than-human nodes in automated networks, such as the meeting of restaurant staff with delivery riders, Richardson challenges a straightforward understanding of automation, instead providing a theorization of the orchestration of calculation, a performance that she argues is always marked by contingency and thus capable of enabling new networks and configurations.

Lockhart and Marvin's paper examines the political economy, imaginaries, and limits of automated environmentally controlled interior spaces. Drawing on case studies from three different experiments in environmental control in Sheffield, England, the authors demonstrate the limits and contradictions of automating ecological processes, where the reality of the need for human interventions, and the attendant labor entailed and contingencies introduced, contradicts the imaginary of "pure" automation. Their analysis excavates the hidden energy and labor that are central to, but often obfuscated by, the relations of automation. Lockhart and Marvin's empirical analysis demonstrates that the idealized reproduction of conditions of interior climatic stability elides more porous relations between the boundaries of inside and outside, and reveals the flows that transgress these boundaries. 
Drawing into conversation scholarship on automation, political ecology and urban political economy, Lockhart and Marvin challenge the potential for designing utopian "inside" environments as a response to and solution for managing turbulent "outside" environments and the problems of accumulation, reproduction, and ecological control associated with our Anthropocene present. Rather, difficulties abound in the aspiration to utilize technology and robotic automation to create the ideal, controlled interior environment, in response to a challenging, uncontrollable exterior environment. Through the case studies explored, controlled indoor ecologies are exposed as complicated socio-technological-ecological assemblages shot through with contradictions and rarely surrendering to desires of automation, optimization, and simplification.

Lynch's paper moves beyond the standard critique of the smart city as an expression of technocapitalism by considering how locally rooted, open sourced digital technology may enable "alternative", counter-capitalist forms of organization of urban life. One of these emergent forms is the technology sovereignty (TS) movement in Barcelona, a decentralized, grassroots network that seeks local, democratic control over the "vital systems and infrastructure of everyday life". Lynch frames this movement within a conceptualization of alternative economic practices and radical relations of care, in which technology - inclusive of infrastructure, code, and data - is viewed as a common good whose governance should be transparent, shared, and democratically administered. Here sovereignty takes as its object the struggle for control over digital information and technological development based on "building community-based technological systems and services with social objectives." In the specific context of technological sovereignty in Barcelona, activists trace a direct link to the food sovereignty movement and other local movements to reclaim control over production and distribution systems vital for everyday life. Here the logics of sovereignty are conceptualized as distinct from in juxtaposition to logics of securitization, which have served to erode, rather than amplify community control over vital systems.

Lynch advances theorization at the intersection of the smart city and processes of digitalization to engage a prefigurative politics of urban geography. One that highlights the possibilities and potentials for the emergence of alternative, counter-hegemonic socio-technical relations in the context of algorithmic life in a contemporary smart city. Lynch's introduction and formulation of technological sovereignty provides a specific language and set of relational practices for challenging the private sector's continued dominance in digital technology development and implementation. This innovation not only challenges traditional smart city approaches to algorithmic urban governance, but presents a vision for an alternative post-capitalist digital future that reimagines socio-spatial and political-economic arrangements of work, property, production, and consumption in the urban sphere. 
Gerhardt's paper uses the case of an emergent, technologically mediated peer-to-peer (P2P) economy and Collaborative Commons movement to make the case for a "non-flat" (i.e. translocal) ontological outlook within anarchist approaches to achieving a post-capitalist society. Open sourced, peer-to-peer digital code and content are here hypothesized as facilitating diminishing marginal costs, giving rise to a nascent, counter-capitalist mode of production. Gerhardt argues that for such a mode of production to blossom requires addressing the emergent, geographically expansive forces that manage to reign in the many prefigurative, counter-capitalist practices that the peer-to-peer economy and movement give rise to. In this intervention, Gerhardt draws on the alternative economic examples of "faircopy," a community centered copyleft arrangement, and "faircoin," a counter-capitalist crypto currency, to illustrate efforts that recognize the need to tackle macro-level assemblages. These cases present a vision for a transition to a postcapitalist world that centers an alternative, collaborative mode of production, where fair reciprocity arrangements replace extractive and exploitative relations and commitments to use value displace exchange value priorities.

Gerhardt also invokes the concept of assemblage, here drawing on Deleuze and Guattari's formulation, in his approach to understanding technology's role in producing, resisting, and transgressing socio-spatial relations. The assemblage framework, he argues, allows for taking "into account the malleable agency of technology", rather than the more common approaches that either ignore it or see it as "overly rigid in its positive or negative causality." Thus technology, understood through this lens of flexibility and malleability, poses possibilities for subversion, adaptation, cooptation, and mutualism that are generative of alternative presents and futures beyond the logics of capitalism.

\section{Conclusion}

This special issue aims to broaden the way scholars theorize and empirically treat the increasingly complex relationships between robots and social life, especially in the context of our historically anthropocentric human geographies. The authors of this special issue engage a range of diverse epistemological, ontological, and methodological commitments, but all in some way address the power dynamics and shifting political economies involved in human-robotic interactions as well as possibilities for resisting and overcoming particular forms of domination and oppression. At the same time, the papers present new avenues for conceptualizing the rise of robots and robotics and the everyday socio-spatial relations of contemporary algorithmic life. In a rapidly evolving present and future, where life is increasingly managed in relation to algorithmic imaginaries and automated fantasies, these papers demonstrate the potential for geographers to make significant interventions and contributions to reveal the limits, contradictions, and messy contingencies of socio-technical assemblages, to trace the shifting spatialities and temporalities of the geographies of algorithmic governance, and to envision radical democratic, post-capitalist, 
emancipatory alternatives. These futures are unlikely to be "robot free," so the question remains how will we build a future set of geographies that acknowledges this reality while also claiming space for the diverse and rich expansion of all forms of life, but human and non-human. 


\section{Bibliography}

Adams W M (2017) Geographies of conservation II: Technology, surveillance and conservation by algorithm. Progress in Human Geography 43(2):337-350

Adams P (2017) Geographies of media and communication I: Metaphysics of encounter. Progress in Human Geography 41(3):365-374

Amoore L (2018) Cloud geographies: Computing, data, sovereignty. Progress in Human Geography 42(1):4-24

Amoore $L$ (2019) Doubt and the algorithm: On the partial accounts of machine learning. Theory, Culture \& Society 36(6):147-169

Amoore L and Raley R (2016) Securing with algorithms: Knowledge, decision, sovereignty. Security Dialogue 48(1):3-10

Arts K, van der Wal R, and Adams W M (2015) Digital technology and the conservation of nature. Ambio 44:661-673

Ash J, Kitchin R and Leszczynski A (Eds). 2018. Digital Geographies. London: Sage

Bakker K and Ritts M (2018) Smart Earth: A meta-review and implications for environmental governance. Global Environmental Change 52:201-211

Batty M (2013) Big data, smart cities and city planning. Dialogues in Human Geography 3(3):274-279. DOI: 10.1177/2043820613513390

Bauwens M, Kostakis V, Pazaitis A (2019) Peer to Peer - A Commons Manifesto. London: University of Westminster Press

Bissell D and Del Casino V J, Jr (2017) Whither labor geography and the rise of the robots? Social and Cultural Geography 18(3):435-442

Benjamin R( Ed.) (2019) Captivating Technology. Race, Carceral Technoscience, and Liberatory Imagination in Everyday Life. Durham: Duke University Press

Boucquey N, Fairbanks L, St. Martin K, Campbell L M, and Wise S (2019). Ocean data portals: Performing a new infrastructure for ocean governance. Environment and Planning D: Society and Space 37(3):484-503 
Boyce G A (2016) The rugged border: Surveillance, policing, and the dynamic materiality of the US/Mexico frontier. Environment and Planning D: Society and Space 34(2):245-262. DOI:

$10.1177 / 0263775815611423$

Brand S (1985) Discussions from the hacker's conference, November 1984. Whole Earth Review 46 (May):44-55

Cockayne D G and Richardson L (2017) Queering code/space: The co-production of socio-sexual codes and digital technologies. Gender, Place \& Culture 24(11):1642-1658

Cockayne D G and Richardson L (2019) The queer times of internet infrastructure and digital systems. In Nash C and Gorman-Murray A (eds) The Geographies of Digital Sexuality (pp 11-27). Singapore: Palgrave Macmillan

Crampton J W (2016) Assemblage of the Vertical: Commercial Drones and Algorithmic Life. Geographica Helvetica 71:137-146. doi:10.5194/gh-71-137-2016

Crampton J W (2019) Platform Biometrics. Surveillance \& Society 17(1-2):54-62. doi: $10.24908 /$ ss.v17i1/2.13111

Crampton J W and Miller A (Eds) (2017) Algorithmic governance-Introduction. Intervention for AntipodeFoundation. http://wp.me/p16RPC-1xG

Datta A (2015) New urban utopias of postcolonial India: 'Entrepreneurial urbanization' in Dholera smart city, Gujarat. Dialogues in Human Geography 5(1):3-22. DOI:

$10.1177 / 2043820614565748$.

Del Casino V J, Jr (2016) Social geographies II: Robots. Progress in Human Geography 40(6):846855

Deleuze G (1992) Postscript on the Societies of Control. October 59 (Winter):3-7

Faroohar R (2019) Don't be Evil. How Big Tech Betrayed its Founding Principles and All of Us. New York: Penguin Random House

Gabrys J (2019) Sensors and sensing practices: Reworking experience across entities, environments, and technologies. Science, Technology, and Human Values 44(5):723-736.

Gieseking J J (2018) Messing with the Attractiveness Algorithm: a Response to Queering Code/Space. Gender, Place and Culture doi:10.1080/0966369X.2017.1379955. 
Gillespie T (2014) The Relevance of Algorithms. In Gillespie T, Boczkowski P J, and Foot K A (eds) Media Technologies (pp 167-193). Cambridge, MA: MIT Press

Greenberg J (2012) This Machine Kills Secrets. How WikiLeakers, Cypherpunks, and Hacktivists Aim to Free the World's Information. New York: Dutton

Harvey D (2003) The new imperialism. New York: Oxford University Press

Holloway L (2007) Subjecting cows to robots: Farming technologies and the making of animal subjects. Environment and Planning D: Society and Space 25(6):1041-1060

Klauser F (2018) Surveillance farm: Towards a research agenda on big data agriculture. Surveillance \& Society 16(3):370-378

Kear M (2017) Playing the credit score game: algorithms, 'positive' data and the personification of financial objects Economy and Society 46 (3-4): 346-368

Kleiner D (2010) The Telekommunist Manifesto. Amsterdam: Institute of Network Cultures

Lehman J (2016) A sea of potential: The politics of global ocean observations. Political Geography 55:113-123

Lehman J (2018) From ships to robots: The social relations of sensing the world ocean. Social Studies of Science 48(1):57-79

Leszczynski A (2019) Glitchy vignettes of platform urbanism. Environment and Planning D: Society and Space. https://doi.org/10.1177/0263775819878721

Linder, T (2019) Surveillance Capitalism and Platform Policing: The Surveillant Assemblage-as-aService. Surveillance \& Society 17(1/2):76-82

Lupton D (2018) How do data come to matter? Living and becoming with personal data. Big Data \& Society $5(2)$

Lynch C R and Del Casino V J, Jr (2019) Smart spaces, information processing, and the question of intelligence. Annals of the American Association of Geographers https://doi.org/10.1080/24694452.2019.1617103 
Macrorie R, Marvin S, and While A (2019) Robotics and automation in the city: A research agenda. Urban Geography. doi: 10.1080/02723638.2019.1698868

Mason P (2015) PostCapitalism: A Guide to our Future. London: Penguin

McConchie A (2015) Hacker cartography: Crowdsourced geography, openstreetmap, and the hacker political imaginary Acme 14 (3):874-898

McDuie-Ra D and Gulson K (2019) The backroads of Al: The uneven geographies of artificial intelligence and development. Area (Online First) https://doi.org/10.1111/area.12602

Miller A (2017) Protocological Violence and the Colonial Database. Intervention for Antipode Foundation. https://wp.me/p16RPC-1xG

Oswald C, Rinner C, and Robinson A (2019) Applications of 3D Printing in Physical Geography Education and Urban Visualization Cartographica: The International Journal for Geographic Information and Geovisualization 54(4): 278-287

Pink S, Sumartojo S, Lupton D, and La Bond C H (2017) Mundane data: The routines, contingencies and accomplishments of digital living. Big Data and Society 4(1):1-12

Richardson L and Bissell D (2019) Geographies of digital skill. Geoforum 99:278-286

Rifkin J (2014) The Zero Marginal Cost Society: The Internet of Things, the Collaborative Commons, and the Eclipse of Capitalism. New York: Palgrave Macmillan

Robbins P (2017) We need to talk about robots. Undisciplined Environments, July 17, 2018. https://undisciplinedenvironments.org/2018/07/17/we-need-to-talk-about-robots/

Rouvroy A and Berns T (2013) Algorithmic governmentality and prospects of emancipation. Disparateness as a precondition for individuation through relationships? Réseaux 177:163-196. DOI : 10.3917/res.177.0163

Shaw I G R (2013) Predator empire: The geopolitics of US drone warfare. Geopolitics 18:536559. DOI: 10.1080/14650045.2012.749241

Shaw I G R (2016) Scorched Atmospheres: The Violent Geographies of the Vietnam War and the Rise of Drone Warfare, Annals of the American Association of Geographers 106(3):688-704. DOI: $10.1080 / 00045608.2015 .1115333$ 
Shaw I G R (2017) Robot Wars: US Empire and geopolitics in the robotic age Security Dialogues 48(5):451-470

Sieber R E, Robinson P J, Johnson P A, and Corbett J M (2016). Doing Public Participation on the Geospatial Web. Annals of the American Association of Geographers 106(5):1030-1046. doi: 10.1080/24694452.2016.1191325

Winner L (1980) Do Artifacts have Politics? Daedalus 109(1):121-136

Wilson M and Kleiner D (2013) Luddite cybercommunism: An email exchange. Anarchist Studies 21(3):73-84

Zook M (2018) Information flows, global finance, and new digital spaces. The New Oxford Handbook of Economic Geography (pp. 575-590).

Zook M and Blankenship J (2018) New spaces of disruption? The failures of Bitcoin and the rhetorical power of algorithmic governance. Geoforum 96:248-255. doi:

10.1016/j.geoforum.2018.08.023. 